\title{
Higher Dimensional Irreducible Representations of the Pure Braid Group
}

\author{
Mohammad N. Abdulrahim(Corresponding author) \\ Department of Mathematics, Beirut Arab University \\ P.O. Box: 11-5020, Beirut, Lebanon \\ E-mail:mna@bau.edu.lb \\ Hassan A. Tarraf \\ Department of Mathematics, Beirut Arab University \\ P.O. Box: 11-5020, Beirut, Lebanon \\ E-mail: hat012@bau.edu.lb
}

Received: May 9, 2011 Accepted: May 24, 2011 Published: November 1, 2011

doi:10.5539/jmr.v3n4p141 URL: http://dx.doi.org/10.5539/jmr.v3n4p141

\begin{abstract}
The reduced Gassner representation is a multi-parameter representation of $P_{n}$, the pure braid group on $\mathrm{n}$ strings. Specializing the parameters $t_{1}, t_{2}, \ldots, t_{n}$ to nonzero complex numbers $x_{1}, x_{2}, \ldots, x_{n}$ gives a representation $G_{n}\left(x_{1}, \ldots, x_{n}\right): P_{n} \rightarrow$ $G L\left(\mathbb{C}^{n-1}\right)$ which is irreducible if and only if $x_{1} \ldots x_{n} \neq 1$. In a previous work, we found a sufficient condition for the irreducibility of the tensor product of two irreducible Gassner representations. In our current work, we find a sufficient condition that guarantees the irreducibility of the tensor product of three Gassner representations. Next, a generalization of our result is given by considering the irreducibility of the tensor product of $k$ representations $(k \geq 3)$.
\end{abstract}

Keywords: Pure braid group, Gassner representation, Irreducible

\section{Introduction}

The pure braid group, $P_{n}$, is a normal subgroup of the braid group, $B_{n}$, on $n$ strings. It has a lot of linear representations. One of them is the Gassner representation which comes from the embedding $P_{n} \rightarrow \operatorname{Aut}\left(F_{n}\right)$, by means of Magnus representation. According to Artin, the automorphism corresponding to the braid generator $\sigma_{i}$ takes $x_{i}$ to $x_{i} x_{i+1} x_{i}^{-1}, x_{i+1}$ to $x_{i}$ and fixes all other free generators. Applying this standard Artin representation to the generators of the pure braid group, we get a representation of the pure braid group by automorphisms. Such a representation has a composition factor, the reduced Gassner representation $G_{n}\left(t_{1}, \ldots, t_{n}\right): P_{n} \rightarrow G L_{n-1}\left(\mathbb{C}\left[t_{1}^{ \pm 1}, \ldots, t_{n}^{ \pm 1}\right]\right)$, where $t_{1}, \ldots, t_{n}$ are indeterminates. We specialize the indeterminates $t_{1}, \ldots, t_{n}$ to nonzero complex numbers $x_{1}, \ldots, x_{n}$ and we define a representation $G_{n}\left(x_{1}, \ldots, x_{n}\right): P_{n} \rightarrow G L_{n-1}(\mathbb{C})=G L\left(\mathbb{C}^{n-1}\right)$ which is irreducible if and only if $x_{1} \ldots x_{n} \neq 1$.

In section 2 of our work, we define the Gassner representation of a free normal subgroup of the pure braid group of rank $n-1$ denoted by $U_{r}$ where $1 \leq r \leq n$. We consider $\mathbb{C}\left[U_{r}\right]$ to be the group algebra of $U_{r}$ over $\mathbb{C}$, and let $\mathcal{A}$ be the augmentation ideal of $\mathbb{C}\left[U_{r}\right]$. On the other hand, if $M$ is any $P_{n}$-module, then $\mathcal{A} M$ is a $P_{n}$-submodule of $M$. We first show that if $\mathbb{C}^{n-1}$ is made into a $P_{n}$-module via the specialization of the reduced Gassner representation $G_{n}\left(x_{1}, \ldots, x_{n}\right): U_{n} \rightarrow$ $G L\left(\mathbb{C}^{n-1}\right)$, then $\mathcal{A} \mathbb{C}^{n-1}$ is its unique minimal nonzero $P_{n}$-submodule. Of course $\mathcal{A} \mathbb{C}^{n-1}=\mathbb{C}^{n-1}$ when $G_{n}\left(x_{1}, \ldots, x_{n}\right)$ is irreducible.

Our objective is to find sufficient conditions that guarantee the irreducibility of the tensor product of $k$ irreducible representations:

$$
G_{n}^{(1)}\left(x_{11}, \ldots, x_{n 1}\right) \otimes \ldots \otimes G_{n}^{(k)}\left(x_{1 k}, \ldots, x_{n k}\right): P_{n} \rightarrow G L\left(\mathbb{C}^{n-1} \otimes \ldots \otimes \mathbb{C}^{n-1}\right)
$$

Here $G_{n}^{(m)}\left(x_{1 m}, \ldots, x_{n m}\right)$ denotes the complex specialization of the reduced Gassner representation of $P_{n}$, where $x_{1 m}, \ldots, x_{n m} \in \mathbb{C}-\{0,1\}$ and $1 \leq m \leq k$.

The case $k=2$ was handled in (Abdulrahim, 2009) and a sufficient condition for the irreducibility of the tensor product was determined. Shortly after, we improved the result (Abdulrahim, 2010). 
In section 3, we deal with the case $k=3$. Our main result is Theorem 1 that states that for $n \geq 3$ and $x=\left(x_{1}, \ldots, x_{n}\right), y=$ $\left(y_{1}, \ldots, y_{n}\right), z=\left(z_{1}, \ldots, z_{n}\right)$, the representation $G_{n}^{(1)}(x) \otimes G_{n}^{(2)}(y) \otimes G_{n}^{(3)}(z): P_{n} \rightarrow G L\left(\mathbb{C}^{n-1} \otimes \mathbb{C}^{n-1} \otimes \mathbb{C}^{n-1}\right)$ is irreducible if there exist some integers $i, j \in\{1, \ldots, n\}$ with $i \neq j$ such that $x_{i} x_{j} \neq y_{i} y_{j}, x_{i} x_{j} \neq z_{i} z_{j}, y_{i} y_{j} \neq z_{i} z_{j}, x_{i} x_{j} y_{i} y_{j} \neq 1, x_{i} x_{j} z_{i} z_{j} \neq$ $1, y_{i} y_{j} z_{i} z_{j} \neq 1, x_{i} x_{j} \neq y_{i} y_{j} z_{i} z_{j}, y_{i} y_{j} \neq x_{i} x_{j} z_{i} z_{j}, z_{i} z_{j} \neq x_{i} x_{j} y_{i} y_{j}, x_{i} x_{j} y_{i} y_{j} z_{i} z_{j} \neq 1, x_{i} \neq x_{j}, y_{i} \neq y_{j}$ and $z_{i} \neq z_{j}$.

In section 4 , we generalize our result to include all values $k \geq 3$. The proof, in the general case, is almost the same as in the case $k=3$. However, we expect the computations to be rather more difficult.

\section{Notations and Preliminaries}

Notation 1. The pure braid group, $P_{n}$, is defined as the kernel of the homomorphism $B_{n} \rightarrow S_{n}$, defined by $\sigma_{i} \rightarrow$ $(i, i+1), 1 \leq i \leq n-1$. It has the following generators:

$$
A_{i, r}=\sigma_{r-1} \sigma_{r-2} \ldots \sigma_{i+1} \sigma_{i}^{2} \sigma_{i+1}^{-1} \ldots \sigma_{r-2}^{-1} \sigma_{r-1}^{-1}, 1 \leq i<r \leq n
$$

We will construct for each $r=1, \ldots, n$ a free normal subgroup of rank $n-1$, namely, $U_{r}$. Let $U_{r}$ be the subgroup generated by the elements

$$
A_{1, r}, A_{2, r}, \ldots, A_{r-1, r}, A_{r, r+1}, \ldots, A_{r, n}
$$

where $A_{i, r}$ are those generators of $P_{n}$ that become trivial after the deletion of the $r$-th strand. For a fixed value of $r$, the image of $A_{i, r}$ under the reduced Gassner representation is denoted by $\tau_{i, r}$, where $\tau_{i, r}=I-P_{i, r} Q_{i, r}$. In other words, the generators of $U_{r}$ are $A_{i, r}$ where $A_{i, r}=A_{r, i}$ whenever $i>r$. It is known that $U_{r}$ generates a free subgroup of $P_{n}$ which is isomorphic to the subgroup $U_{n}$ freely generated by $\left\{A_{1, n}, A_{2, n}, \ldots, A_{n-1, n}\right\}$. This is intuitively clear because it is quite arbitrary how we assign indices to the braid "strings". For more details, see (Birman, 1975).

For simplicity, we denote $A_{i, r}$ by $\tau_{i, r}$. That is, we have

$$
\tau_{1, r}=A_{1, r}, \ldots, \tau_{r-1, r}=A_{r-1, r}, \tau_{r+1, r}=A_{r, r+1}, \tau_{r+2, r}=A_{r, r+2}, \ldots, \tau_{n, r}=A_{r, n}
$$

Definition 1. The reduced Gassner representation restricted to $U_{r}$ is defined as follows: $\tau_{i, r}=I-P_{i, r} Q_{i, r}$ for $1 \leq i, r \leq n$. For $i<r, P_{i, r}$ is the column vector given by:

$$
(1-t_{1}, \ldots, 1-t_{i-1}, \underbrace{1-t_{i} t_{r}}_{i}, t_{r}\left(1-t_{i+1}\right), \ldots, t_{r}\left(1-t_{r-1}\right), \underbrace{t_{r+1}-1}_{r}, t_{r+2}-1, \ldots, t_{n}-1)^{T},
$$

and for $n \geq i>r, P_{i, r}$ is the column vector given by:

$$
(t_{r}\left(t_{1}-1\right), \ldots, t_{r}\left(t_{r-1}-1\right), \underbrace{1-t_{r+1}, \ldots, 1-t_{i}}_{i-r}, 1-t_{i+1} t_{r}, t_{r}\left(1-t_{i+2}\right), \ldots, t_{r}\left(1-t_{n}\right))^{T} .
$$

Here $T$ is the transpose and $Q_{i, r}$ is the row vector given by:

$$
Q_{i, r}=(0, \ldots, 0, \underbrace{1}_{i}, 0, \ldots, 0), \quad 1 \leq i, r \leq n
$$

The definition of the reduced Gassner representation restricted to a free normal subgroup is the same, up to equivalence. Representations given by pseudoreflections $I-A_{i} B_{i}$ and $I-C_{i} D_{i}$ are equivalent if the inner products $\left(B_{i} A_{j}\right)$ and $\left(D_{i} C_{j}\right)$ are conjugate by a diagonal matrix. Here, $A_{i}, C_{i}$ are column vectors and $B_{i}, D_{i}$ are row vectors.

We identify $\mathbb{C}^{n-1}$ with $(n-1) \times 1$ column vectors. We let $e_{1}, \ldots, e_{n-1}$ denote the standard basis for $\mathbb{C}^{n-1}$, and we consider matrices to act by left multiplication on column vectors.

Definition 2. If $r=a_{1} e_{1}+\cdots+a_{n-1} e_{n-1} \in \mathbb{C}^{n-1}$, the support of $r$, denoted supp(r), is the set $\left\{e_{i} \mid a_{i} \neq 0\right\}$. If $s=\Sigma a_{i j k}\left(e_{i} \otimes e_{j} \otimes e_{k}\right) \in \mathbb{C}^{n-1} \otimes \mathbb{C}^{n-1} \otimes \mathbb{C}^{n-1}$, the support of $s$, also denoted $\operatorname{supp}(s)$, is the set $\left\{e_{i} \otimes e_{j} \otimes e_{k} \mid a_{i j k} \neq 0\right\}$, and $a_{i j k}$ is called the coefficient of $e_{i} \otimes e_{j} \otimes e_{k}$ in $s$.

Definition 3. Given an integer $r, 1 \leq r \leq n$ and a vector $t=\left(t_{1}, \ldots, t_{n}\right)$. We define $v_{i, r}(t)=e_{i}-\tau_{i, r}(t)\left(e_{i}\right)=\left(I-\tau_{i, r}(t)\right)\left(e_{i}\right)$. In other words, we have the following: 
For $1 \leq i \leq r-1, v_{i, r}(t)=$

$$
(1-t_{1}, \ldots, 1-t_{i-1}, \underbrace{1-t_{i} t_{r}}_{i}, t_{r}\left(1-t_{i+1}\right), \ldots, t_{r}\left(1-t_{r-1}\right), \underbrace{t_{r+1}-1}_{r}, t_{r+2}-1, \ldots, t_{n}-1)^{T}
$$

and for $n \geq i>r, v_{i, r}(t)=$

$$
(t_{r}\left(t_{1}-1\right), \ldots, t_{r}\left(t_{r-1}-1\right), \underbrace{1-t_{r+1}, \ldots, 1-t_{i}}_{i-r}, 1-t_{i+1} t_{r}, t_{r}\left(1-t_{i+2}\right), \ldots, t_{r}\left(1-t_{n}\right))^{T}
$$

Lemma 1. For $t=\left(t_{1}, \ldots, t_{n}\right)$, we have:

$$
\begin{array}{cl}
\text { (1) } \tau_{i, r}(t)\left(v_{i, s}(t)\right)=v_{i, s}(t)+\left(t_{i} t_{s}-1\right) v_{i, r}(t) & \text { for } 1 \leq i \leq s-1, \\
\tau_{i, r}(t)\left(v_{i, s}(t)\right)=v_{i, s}(t)+\left(t_{i+1} t_{s}-1\right) v_{i, r}(t) & \text { for } 1 \leq s<i, \\
\text { (2) } \tau_{i, r}(t)\left(v_{j, s}(t)\right)=v_{j, s}(t)+\left(t_{i}-1\right) v_{i, r}(t) & \text { for } i<j<s, \\
\tau_{i, r}(t)\left(v_{j, s}(t)\right)=v_{j, s}(t)+t_{s}\left(t_{i}-1\right) v_{i, r}(t) & \text { for } \quad j<i<s, \\
\tau_{i, r}(t)\left(v_{j, s}(t)\right)=v_{j, s}(t)+\left(1-t_{i+1}\right) v_{i, r}(t) & \text { for } \quad j<s<i, \\
\text { (3) } \tau_{i, r}(t)\left(v_{j, s}(t)\right)=v_{j, s}(t)+t_{s}\left(1-t_{i}\right) v_{i, r}(t) & \text { for } i<s<j, \\
\tau_{i, r}(t)\left(v_{j, s}(t)\right)=v_{j, s}(t)+\left(t_{i+1}-1\right) v_{i, r}(t) & \text { for } \quad s<i<j, \\
\tau_{i, r}(t)\left(v_{j, s}(t)\right)=v_{j, s}(t)+t_{s}\left(t_{i+1}-1\right) v_{i, r}(t) & \text { for } \quad s<j<i .
\end{array}
$$

For a fixed value of $r$, we use this Lemma to determine elements in the group algbera $\mathbb{C}\left(P_{n}\right)$ over $\mathbb{C}$ that send the vector $v_{i, r}$ to the vector $v_{i+1, r}$ and other elements that send the vector $v_{i, r}$ to $v_{i-1, r}$.

Definition 4. Given an integer $r$ such that $1 \leq r \leq n$. Consider the following elements of the pure braid group algebra:

$f_{i, r}=\left\{\begin{array}{l}\tau_{i, r}-\left(t_{i} t_{r}\right) \tau_{i+1, r}, \quad 1 \leq i<r-1 \\ \tau_{i, r}-\left(t_{i} t_{r}\right) \tau_{i+2, r}, \quad i=r-1 \\ \tau_{i, r}-\left(t_{i+1} t_{r}\right) \tau_{i+1, r}, \quad 1 \leq r<i \leq n-1\end{array}\right.$

and

$g_{i, r}=\left\{\begin{array}{l}\tau_{i, r}-\left(t_{i} t_{r}\right) \tau_{i-1, r}, \quad 1 \leq i \leq r-1 \\ \tau_{i, r}-\left(t_{i+1} t_{r}\right) \tau_{i-2, r}, \quad i=r+1 \leq n-1 \\ \tau_{i, r}-\left(t_{i+1} t_{r}\right) \tau_{i-1, r}, \quad r+1<i \leq n-1\end{array}\right.$

Lemma 2. Fix an integer $r, 1 \leq r \leq n$. For all integers $i, 1 \leq i \leq n-1$, the action of the elements of the pure braid group algebra, namely, $f_{i, r}$ and $g_{i, r}$, on the vectors $v_{i, r}$ is given by:

(i) $f_{i, r}\left(v_{i, r}\right)=\left\{\begin{array}{l}-t_{i} t_{r}^{2}\left(t_{i+1}-1\right) v_{i+1, r}, \quad 1 \leq i<r-1 \\ -t_{r-1} t_{r}\left(1-t_{r+2}\right) v_{r+1, r}, \quad i=r-1 \leq n-3 \\ -t_{i+1} t_{r}^{2}\left(t_{i+2}-1\right) v_{i+1, r}, 1 \leq r<i \leq n-2\end{array}\right.$

and

(ii) $g_{i, r}\left(v_{i, r}\right)=\left\{\begin{array}{l}-t_{i} t_{r}\left(t_{i-1}-1\right) v_{i-1, r}, 1 \leq i \leq r-1 \\ -t_{r+2} t_{r}^{2}\left(1-t_{r-1}\right) v_{r-1, r}, \quad i=r+1 \leq n-1 \\ -t_{i+1} t_{r}\left(t_{i}-1\right) v_{i-1, r}, r+1<i \leq n-1 .\end{array}\right.$

Notation 2. Let $G_{n}\left(x_{1}, \ldots, x_{n}\right)$ denote the reduced Gassner representation of $P_{n}$ under the specialization $t_{i} \rightarrow x_{i}$, where $x_{i}$ is a non-zero complex number.

Lemma 3. Having $U_{r}$ a free normal subgroup of the pure braid group, we let $G_{n}\left(x_{1}, \ldots, x_{n}\right): U_{r} \rightarrow G L\left(\mathbb{C}^{n-1}\right)$ be $a$ specialization of the reduced Gassner representation restricted to $U_{r}$ making $\mathbb{C}^{n-1}$ into a $U_{r}$-module, where $n \geq 3$. Then

(a) Let $\mathcal{A}$ be the kernel of the homomorphism $\mathbb{C}\left[U_{r}\right] \rightarrow \mathbb{C}$ induced by $\tau_{i, r} \rightarrow 1$ (the augmentation ideal). Let $x$ be the vector $\left(x_{1}, \ldots, x_{n}\right)$.Then $\mathcal{A} \mathbb{C}^{n-1}$ is equal to the $\mathbb{C}$-vector space spanned by $v_{1, r}(x), \ldots, v_{r-1, r}(x), v_{r+1, r}(x), \ldots, v_{n, r}(x)$.

(b) If $M$ is a nonzero $U_{r}$-submodule of $\mathbb{C}^{n-1}$, then $\mathcal{A} \mathbb{C}^{n-1} \subseteq M$. Hence $\mathcal{A} \mathbb{C}^{n-1}$ is the unique minimal nonzero $U_{r}$-submodule of $\mathbb{C}^{n-1}$. 
(c) If $p\left(x_{1}, \ldots, x_{n}\right)=\left(x_{r}-1\right)^{n-2}\left(x_{1} x_{2} \ldots x_{n}-1\right) \neq 0$, then $\mathcal{A} \mathbb{C}^{n-1}=\mathbb{C}^{n-1}$, and $G_{n}\left(x_{1}, x_{2}, \ldots, x_{n}\right)$ restricted to $U_{r}$ is irreducible.

Proof. Here, we will take the free normal subgroup, $U_{r}$, of rank $n-1$. Notice that, in the proof of (b), we need the fact that if $v_{j, r} \in M$ for some $j$ and $r$ then all $v_{i, r} \in M$. This is due to Lemma 1. As for (c), the determinant of the matrix, whose columns are the vectors $v_{1, r}(x), \ldots, v_{n, r}(x)$, is $p(x)=\left(x_{r}-1\right)^{n-2}\left(x_{1} x_{2} \ldots x_{n}-1\right)$, so if $p(x) \neq 0$ then $v_{1, r}(x), \ldots v_{n, r}(x)$ is a basis for $\mathbb{C}^{n-1}$ and $\mathcal{A} \mathbb{C}^{n-1}=\mathbb{C}^{n-1}$.

Hence, $\mathcal{A} \mathbb{C}^{n-1}$ is its unique minimal nonzero $U_{r}$-submodule. Of course $\mathcal{A} \mathbb{C}^{n-1}=\mathbb{C}^{n-1}$ when $G_{n}\left(x_{1}, \ldots, x_{n}\right)$ is irreducible. For more details, see (Formanek, 1996) and (Abdulrahim, 2005).

\section{The Tensor Product of Three Irreducible Representations}

For $1 \leq j \leq n$, we consider the normal subgroup of rank $n-1$, namely, $U_{j}$, defined as before. We find a sufficient condition for the irreducibility of the tensor product of three irreducible representations of $U_{j}$ :

$$
\begin{gathered}
G_{n}^{(1)}\left(x_{11}, \ldots, x_{n 1}\right) \otimes G_{n}^{(2)}\left(x_{12}, \ldots, x_{n 2}\right) \otimes G_{n}^{(3)}\left(x_{13}, \ldots, x_{n 3}\right): \\
U_{j} \rightarrow G L\left(\mathbb{C}^{n-1} \otimes \mathbb{C}^{n-1} \otimes \mathbb{C}^{n-1}\right)
\end{gathered}
$$

We now introduce Proposition 1 that provides us with a sufficient condition for irreducibility. For simplicity, we write

$$
x_{u v}= \begin{cases}x_{u} & \text { if } v=1 \\ y_{u} & \text { if } v=2 \\ z_{u} & \text { if } v=3\end{cases}
$$

for $1 \leq u \leq n$.

Proposition 1. Suppose that $x=\left(x_{1}, \ldots, x_{n}\right), y=\left(y_{1}, \ldots, y_{n}\right)$ and $z=\left(z_{1}, \ldots, z_{n}\right)$

$\in \mathbb{C}^{n}$, where $x_{s}, y_{s}, z_{s} \in \mathbb{C}-\{0,1\}$ for $1 \leq s \leq n$. Suppose also that for some $i<j$, we have that

$$
\begin{gathered}
x_{i} x_{j} \neq y_{i} y_{j}, x_{i} x_{j} \neq z_{i} z_{j}, y_{i} y_{j} \neq z_{i} z_{j}, x_{i} x_{j} y_{i} y_{j} \neq 1, x_{i} x_{j} z_{i} z_{j} \neq 1, y_{i} y_{j} z_{i} z_{j} \neq 1, \\
x_{i} x_{j} \neq y_{i} y_{j} z_{i} z_{j}, y_{i} y_{j} \neq x_{i} x_{j} z_{i} z_{j}, z_{i} z_{j} \neq x_{i} x_{j} y_{i} y_{j}, x_{i} x_{j} y_{i} y_{j} z_{i} z_{j} \neq 1, \\
x_{i} \neq x_{j}, y_{i} \neq y_{j}, z_{i} \neq z_{j} .
\end{gathered}
$$

Let $M$ be a nonzero $U_{j}$-submodule of $\mathbb{C}^{n-1} \otimes \mathbb{C}^{n-1} \otimes \mathbb{C}^{n-1}$ under the action of $G_{n}^{(1)}(x) \otimes G_{n}^{(2)}(y) \otimes G_{n}^{(3)}(z): U_{j} \rightarrow$ $G L\left(\mathbb{C}^{n-1} \otimes \mathbb{C}^{n-1} \otimes \mathbb{C}^{n-1}\right)$, where $n \geq 3$. For simplicity, we write $v_{p, j}=v_{p}$ for $p \in\{1, \ldots, j-1, j+1, \ldots, n\}$. Then $M$ contains all $v_{p}(x) \otimes v_{q}(y) \otimes v_{r}(z)$ for $p, q, r \in\{1, \ldots, j-1, j+1, \ldots, n\}$. Thus $M$ contains $\mathcal{A} \mathbb{C}^{n-1} \otimes \mathcal{A} \mathbb{C}^{n-1} \otimes \mathcal{A} \mathbb{C}^{n-1}$. Here, the action of $U_{j}$ on the first factor is induced by $G_{n}^{(1)}\left(x_{1}, \ldots, x_{n}\right)$, the action of $U_{j}$ on the second factor is induced by $G_{n}^{(2)}\left(y_{1}, \ldots, y_{n}\right)$ and the action of $U_{j}$ on the third factor is induced by $G_{n}^{(3)}\left(z_{1}, \ldots, z_{n}\right)$.

\section{Proof.}

Claim 1. There exists an $s \in\{1, \ldots, n-1\}$ such that $e_{s} \otimes e_{s} \otimes e_{s} \in \operatorname{supp}(m)$ for some $m \in M$.

Proof of Claim 1.

Case 1. Suppose that there exists an $s \in\{1, \ldots, n-1\}$ such that $e_{s} \otimes e_{s} \otimes e_{s} \in \operatorname{supp}(m)$, then we are done.

Case 2. Suppose that there exists an $s \in\{1, \ldots, n-1\}$, with $s \neq i$, such that at least one of $e_{i} \otimes e_{s} \otimes e_{s}, e_{s} \otimes e_{i} \otimes e_{s}, e_{s} \otimes e_{s} \otimes$ $e_{i}, e_{i} \otimes e_{i} \otimes e_{s}, e_{i} \otimes e_{s} \otimes e_{i}, e_{s} \otimes e_{i} \otimes e_{i} \in \operatorname{supp}(m)$.

We write $m$ as follows:

$m=a e_{i} \otimes e_{s} \otimes e_{s}+b e_{s} \otimes e_{i} \otimes e_{s}+c e_{s} \otimes e_{s} \otimes e_{i}+d e_{i} \otimes e_{i} \otimes e_{s}+e e_{i} \otimes e_{s} \otimes e_{i}+f e_{s} \otimes e_{i} \otimes e_{i}+W$.

Here, at least one of $a, b, c, d, e, f \in \mathbb{C}^{*}$ and $\operatorname{supp}(W)$ does not contain any of $e_{i} \otimes e_{s} \otimes e_{s}, e_{s} \otimes e_{i} \otimes e_{s}, e_{s} \otimes e_{s} \otimes e_{i}, e_{i} \otimes e_{i} \otimes$ $e_{s}, e_{i} \otimes e_{s} \otimes e_{i}, e_{s} \otimes e_{i} \otimes e_{i}$. We also assume that $\operatorname{supp}(W)$ does not contain any of $e_{\alpha} \otimes e_{\alpha} \otimes e_{\alpha}$ for any $\alpha$.

Applying $\tau_{i}$ on $m$, we obtain

$\tau_{i}(m)=a \tau_{i}\left(e_{i}\right) \otimes e_{s} \otimes e_{s}+b e_{s} \otimes \tau_{i}\left(e_{i}\right) \otimes e_{s}+c e_{s} \otimes e_{s} \otimes \tau_{i}\left(e_{i}\right)+d \tau_{i}\left(e_{i}\right) \otimes \tau_{i}\left(e_{i}\right) \otimes e_{s}+$ 


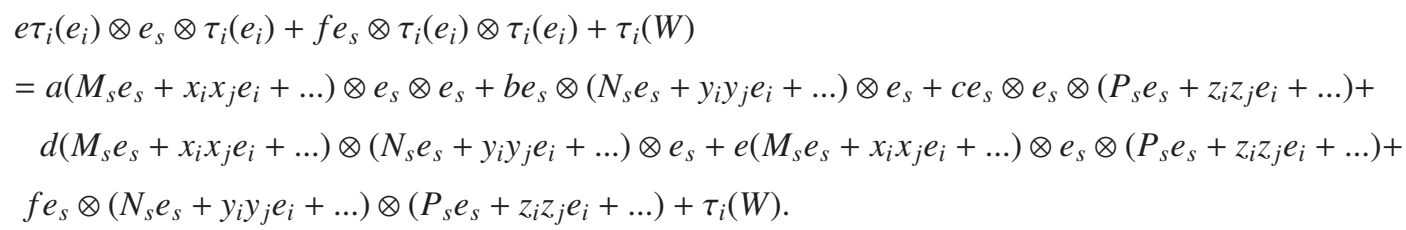

Here, $M_{s}, N_{s}$ and $P_{s}$ are all nonzero complex numbers given as follows:

If $s<i$ then $M_{s}=x_{s}-1, N_{s}=y_{s}-1$ and $P_{s}=z_{s}-1$.

If $i<s<j$ then $M_{s}=x_{j}\left(x_{s}-1\right), N_{s}=y_{j}\left(y_{s}-1\right)$ and $P_{s}=z_{j}\left(z_{s}-1\right)$.

If $s \geq j$ then $M_{s}=1-x_{s}, N_{s}=1-y_{s}$ and $P_{s}=1-z_{s}$.

$\tau_{i}(m)=\left(a M_{s}+b N_{s}+c P_{s}+d M_{s} N_{s}+e M_{s} P_{s}+f N_{s} P_{s}\right) e_{s} \otimes e_{s} \otimes e_{s}+\left(a x_{i} x_{j}+\right.$

$\left.d N_{s} x_{i} x_{j}+e P_{s} x_{i} x_{j}\right) e_{i} \otimes e_{s} \otimes e_{s}+\left(b y_{i} y_{j}+d M_{s} y_{i} y_{j}+f P_{s} y_{i} y_{j}\right) e_{s} \otimes e_{i} \otimes$

$e_{s}+\left(c z_{i} z_{j}+e M_{s} z_{i} z_{j}+f N_{s} z_{i} z_{j}\right) e_{s} \otimes e_{s} \otimes e_{i}+d x_{i} x_{j} y_{i} y_{j} e_{i} \otimes e_{i} \otimes e_{s}+$

$e x_{i} x_{j} z_{i} z_{j} e_{i} \otimes e_{s} \otimes e_{i}+f y_{i} y_{j} z_{i} z_{j} e_{s} \otimes e_{i} \otimes e_{i}+\ldots+\tau_{i}(W)$.

The coefficients of $e_{s} \otimes e_{s} \otimes e_{s}$ in $\tau_{i}(m), \ldots, \tau_{i}^{6}(m)$ are given as follows:

In $\tau_{i}(m)$ : the coefficient of $e_{s} \otimes e_{s} \otimes e_{s}$ is $a M_{s}+b N_{s}+c P_{s}+d M_{s} N_{s}+e M_{s} P_{s}+f N_{s} P_{s}$.

In $\tau_{i}^{2}(m)$ : the coefficients of $e_{s} \otimes e_{s} \otimes e_{s}$ is

$a M_{s}\left(1+x_{i} x_{j}\right)+b N_{s}\left(1+y_{i} y_{j}\right)+c P_{s}\left(1+z_{i} z_{j}\right)+d M_{s} N_{s}\left(1+x_{i} x_{j}\right)\left(1+y_{i} y_{j}\right)+e M_{s} P_{s}\left(1+x_{i} x_{j}\right)\left(1+z_{i} z_{j}\right)+f N_{s} P_{s}\left(1+y_{i} y_{j}\right)\left(1+z_{i} z_{j}\right)$.

In $\tau_{i}^{3}$ : the coefficient of $e_{s} \otimes e_{s} \otimes e_{s}$ is

$a M_{s}\left(1+x_{i} x_{j}+x_{i}^{2} x_{j}^{2}\right)+b N_{s}\left(1+y_{i} y_{j}+y_{i}^{2} y_{j}^{2}\right)+c P_{s}\left(1+z_{i} z_{j}+z_{i}^{2} z_{j}^{2}\right)+d M_{s} N_{s}\left(1+x_{i} x_{j}+x_{i}^{2} x_{j}^{2}\right)\left(1+y_{i} y_{j}+y_{i}^{2} y_{j}^{2}\right)+e M_{s} P_{s}(1+$ $\left.x_{i} x_{j}+x_{i}^{2} x_{j}^{2}\right)\left(1+z_{i} z_{j}+z_{i}^{2} z_{j}^{2}\right)+f N_{s} P_{s}\left(1+y_{i} y_{j}+y_{i}^{2} y_{j}^{2}\right)\left(1+z_{i} z_{j}+z_{i}^{2} z_{j}^{2}\right)$.

Likewise for $\tau_{i}^{4}(m), \tau_{i}^{5}(m)$ and $\tau_{i}^{6}(m)$. Therefore, we consider the $6 \times 6$ matrix whose first column corresponds to the coefficients of $a$, the second column corresponds to the coefficient of $b$ and so on. More precisely, the matrix obtained is given by

$$
A=\left[a_{\alpha \beta}\right]=\left(\begin{array}{cccccc}
a_{11} & a_{12} & a_{13} & a_{11} \cdot a_{12} & a_{11} \cdot a_{13} & a_{12} \cdot a_{13} \\
a_{21} & a_{22} & a_{23} & a_{21} \cdot a_{22} & a_{21} \cdot a_{23} & a_{22} \cdot a_{23} \\
\vdots & \vdots & \vdots & \vdots & \vdots & \vdots \\
a_{61} & a_{62} & a_{63} & a_{61} \cdot a_{62} & a_{61} \cdot a_{63} & a_{62} \cdot a_{63}
\end{array}\right)
$$

Here, $a_{\alpha 1}=M_{s}\left(\sum_{k=0}^{\alpha-1} x_{i}^{k} x_{j}^{k}\right), \quad a_{\alpha 2}=N_{s}\left(\sum_{k=0}^{\alpha-1} y_{i}^{k} y_{j}^{k}\right), \quad a_{\alpha 3}=P_{s}\left(\sum_{k=0}^{\alpha-1} z_{i}^{k} z_{j}^{k}\right), 1 \leq \alpha \leq 6$.

The determinant of the matrix above is

$-M_{s}^{3} N_{s}^{3} P_{s}^{3} x_{i}^{3} x_{j}^{3} y_{i}^{3} y_{j}^{3} z_{i}^{3} z_{j}^{3}\left(x_{i} x_{j}-y_{i} y_{j}\right)^{2}\left(x_{i} x_{j}-z_{i} z_{j}\right)^{2}\left(y_{i} y_{j}-z_{i} z_{j}\right)^{2}\left(x_{i} x_{j} y_{i} y_{j}-1\right)\left(x_{i} x_{j} z_{i} z_{j}-1\right)\left(y_{i} y_{j} z_{i} z_{j}-1\right)\left(x_{i} x_{j}-y_{i} y_{j} z_{i} z_{j}\right)\left(y_{i} y_{j}-\right.$ $\left.x_{i} x_{j} z_{i} z_{j}\right)\left(z_{i} z_{j}-x_{i} x_{j} y_{i} y_{j}\right)$.

Using the hypothesis, we get that the determinant of the matrix $A$ is nonzero. Then, at least one of $\tau_{i}(m), \tau_{i}^{2}(m), \tau_{i}^{3}(m)$, $\tau_{i}^{4}(m), \tau_{i}^{5}(m), \tau_{i}^{6}(m)$ has $e_{s} \otimes e_{s} \otimes e_{s}$ in its support.

Case 3. Suppose that for every $\alpha \in\{1, \ldots, n\}$, we have that $e_{i} \otimes e_{i} \otimes e_{\alpha}, e_{i} \otimes e_{\alpha} \otimes e_{i}, e_{\alpha} \otimes e_{i} \otimes e_{i}, e_{i} \otimes e_{\alpha} \otimes e_{\alpha}, e_{\alpha} \otimes$ $e_{i} \otimes e_{\alpha}, e_{\alpha} \otimes e_{\alpha} \otimes e_{i} \notin \operatorname{supp}(m)$, but there exist exist distinct $\beta \quad \& \quad \gamma$ with $\beta \neq i, \gamma \neq i$ such that at least one of $e_{i} \otimes e_{\beta} \otimes e_{\gamma}, e_{\beta} \otimes e_{i} \otimes e_{\gamma}, e_{\beta} \otimes e_{\gamma} \otimes e_{i}, e_{\beta} \otimes e_{\beta} \otimes e_{\gamma}, e_{\beta} \otimes e_{\gamma} \otimes e_{\beta}, e_{\gamma} \otimes e_{\beta} \otimes e_{\beta} \in \operatorname{supp}(m)$. Here, $i$ is the integer given in the hypothesis of Proposition 1.

Here, $m$ can be written as:

$m=a e_{i} \otimes e_{\beta} \otimes e_{\gamma}+b e_{\beta} \otimes e_{i} \otimes e_{\gamma}+c e_{\beta} \otimes e_{\gamma} \otimes e_{i}+d e_{i} \otimes e_{\gamma} \otimes e_{\beta}+e e_{\gamma} \otimes e_{i} \otimes e_{\beta}+$

$f e_{\gamma} \otimes e_{\beta} \otimes e_{i}+g e_{\beta} \otimes e_{\beta} \otimes e_{\gamma}+h e_{\beta} \otimes e_{\gamma} \otimes e_{\beta}+i e_{\gamma} \otimes e_{\beta} \otimes e_{\beta}+j e_{\gamma} \otimes e_{\gamma} \otimes e_{\beta}+$

$k e_{\gamma} \otimes e_{\beta} \otimes e_{\gamma}+l e_{\beta} \otimes e_{\gamma} \otimes e_{\gamma}+W$.

Here, at least one of $a, b, c, d, e, f, g, h, i, j, k, l \in \mathbb{C}^{*}$ and $\operatorname{supp}(W)$ does not contain any of the previous tensors. We also 
assume that $\operatorname{supp}(W)$ does not contain any of $e_{\alpha} \otimes e_{\alpha} \otimes e_{\alpha}$ for any $\alpha$.

Applying $\tau_{\beta}$ on $m$, we obtain

$$
\begin{aligned}
& \tau_{\beta}(m)=a e_{i} \otimes\left(N_{i} e_{i}+N_{\gamma} e_{\gamma}+y_{\beta} y_{j} e_{\beta}+\ldots\right) \otimes e_{\gamma}+b\left(M_{i} e_{i}+M_{\gamma} e_{\gamma}+x_{\beta} x_{j} e_{\beta}+\ldots\right) \otimes \\
& e_{i} \otimes e_{\gamma}+c\left(M_{i} e_{i}+M_{\gamma} e_{\gamma}+x_{\beta} x_{j} e_{\beta}+\ldots\right) \otimes e_{\gamma} \otimes e_{i}+d e_{i} \otimes e_{\gamma} \otimes\left(P_{i} e_{i}+P_{\gamma} e_{\gamma}\right. \\
& \left.+z_{\beta} z_{j} e_{\beta}+\ldots\right)+e e_{\gamma} \otimes e_{i} \otimes\left(P_{i} e_{i}+P_{\gamma} e_{\gamma}+z_{\beta} z_{j} e_{\beta}+\ldots\right)+f e_{\gamma} \otimes\left(N_{i} e_{i}+N_{\gamma}\right. \\
& \left.e_{\gamma}+y_{\beta} y_{j} e_{\beta}+\ldots\right) \otimes e_{i}+g\left(M_{i} e_{i}+M_{\gamma} e_{\gamma}+x_{\beta} x_{j} e_{\beta}+\ldots\right) \otimes\left(N_{i} e_{i}+N_{\gamma} e_{\gamma}+y_{\beta}\right. \\
& \left.y_{j} e_{\beta}+\ldots\right) \otimes e_{\gamma}+h\left(M_{i} e_{i}+M_{\gamma} e_{\gamma}+x_{\beta} x_{j} e_{\beta}+\ldots\right) \otimes e_{\gamma} \otimes\left(P_{i} e_{i}+P_{\gamma} e_{\gamma}+z_{\beta} z_{j}\right. \\
& \left.e_{\beta}+\ldots\right)+i e_{\gamma} \otimes\left(N_{i} e_{i}+N_{\gamma} e_{\gamma}+y_{\beta} y_{j} e_{\beta}+\ldots\right) \otimes\left(P_{i} e_{i}+P_{\gamma} e_{\gamma}+z_{\beta} z_{j} e_{\beta}+\ldots\right) \\
& +j e_{\gamma} \otimes e_{\gamma} \otimes\left(P_{i} e_{i}+P_{\gamma} e_{\gamma}+z_{\beta} z_{j} e_{\beta}+\ldots\right)+k e_{\gamma} \otimes\left(N_{i} e_{i}+N_{\gamma} e_{\gamma}+y_{\beta} y_{j} e_{\beta}+\right. \\
& \ldots) \otimes e_{\gamma}+l\left(M_{i} e_{i}+M_{\gamma} e_{\gamma}+x_{\beta} x_{j} e_{\beta}+\ldots\right) \otimes e_{\gamma} \otimes e_{\gamma}+\tau_{\beta}(W) \\
& =\left(a N_{i}+b M_{i}+g M_{i} N_{i}\right) e_{i} \otimes e_{i} \otimes e_{\gamma}+\left(a N_{\gamma}+d P_{\gamma}+g M_{i} N_{\gamma}+h M_{i} P_{\gamma}+l M_{i}\right) e_{i} \\
& \otimes e_{\gamma} \otimes e_{\gamma}+\left(b M_{\gamma}+e P_{\gamma}+g M_{\gamma} N_{i}+i N_{i} P_{\gamma}+k N_{i}\right) e_{\gamma} \otimes e_{i} \otimes e_{\gamma}+\left(c M_{i}+d p_{i}\right. \\
& \left.+h M_{i} P_{i}\right) e_{i} \otimes e_{\gamma} \otimes e_{i}+\left(c M_{\gamma}+f N_{\gamma}+h M_{\gamma} P_{i}+i N_{\gamma} P_{i}+j P_{i}\right) e_{\gamma} \otimes e_{\gamma} \otimes e_{i}+ \\
& \left(e P_{i}+f N_{i}+i N_{i} P_{i}\right) e_{\gamma} \otimes e_{i} \otimes e_{i}+\left(g M_{\gamma} N_{\gamma}+h M_{\gamma} P_{\gamma}+i N_{\gamma} P_{\gamma}+j P_{\gamma}+k N_{\gamma}\right. \\
& \left.+l M_{\gamma}\right) e_{\gamma} \otimes e_{\gamma} \otimes e_{\gamma}+a y_{\beta} y_{j} e_{i} \otimes e_{\beta} \otimes e_{\gamma}+b x_{\beta} x_{j} e_{\beta} \otimes e_{i} \otimes e_{\gamma}+c x_{\beta} x_{j} e_{\beta} \otimes e_{\gamma} \\
& \otimes e_{i}+d z_{\beta} z_{j} e_{i} \otimes e_{\gamma} \otimes e_{\beta}+e z_{\beta} z_{j} e_{\gamma} \otimes e_{i} \otimes e_{\beta}+f y_{\beta} y_{j} e_{\gamma} \otimes e_{\beta} \otimes e_{i}+g M_{i} y_{\beta} y_{j} \\
& e_{i} \otimes e_{\beta} \otimes e_{\gamma}+g M_{\gamma} y_{\beta} y_{j} e_{\gamma} \otimes e_{\beta} \otimes e_{\gamma}+g x_{\beta} x_{j} N_{i} e_{\beta} \otimes e_{i} \otimes e_{\gamma}+g x_{\beta} x_{j} N_{\gamma} e_{\beta} \otimes \\
& e_{\gamma} \otimes e_{\gamma}+g x_{\beta} x_{j} y_{\beta} y_{j} e_{\beta} \otimes e_{\beta} \otimes e_{\gamma}+h M_{i} z_{\beta} z_{j} e_{i} \otimes e_{\gamma} \otimes e_{\beta}+h M_{\gamma} z_{\beta} z_{j} e_{\gamma} \otimes e_{\gamma} \\
& \otimes e_{\beta}+h x_{\beta} x_{j} P_{i} e_{\beta} \otimes e_{\gamma} \otimes e_{i}+h x_{\beta} x_{j} P_{\gamma} e_{\beta} \otimes e_{\gamma} \otimes e_{\gamma}+h x_{\beta} x_{j} z_{\beta} z_{j} e_{\beta} \otimes e_{\gamma} \otimes \\
& e_{\beta}+i N_{i} z_{\beta} z_{j} e_{\gamma} \otimes e_{i} \otimes e_{\beta}+i N_{\gamma} z_{\beta} z_{j} e_{\gamma} \otimes e_{\gamma} \otimes e_{\beta}+i y_{\beta} y_{j} P_{i} e_{\gamma} \otimes e_{\beta} \otimes e_{i}+i y_{\beta} \\
& y_{j} P_{\gamma} e_{\gamma} \otimes e_{\beta} \otimes e_{\gamma}+i y_{\beta} y_{j} z_{\beta} z_{j} e_{\gamma} \otimes e_{\beta} \otimes e_{\beta}+j z_{\beta} z_{j} e_{\gamma} \otimes e_{\gamma} \otimes e_{\beta}+k y_{\beta} y_{j} e_{\gamma} \otimes \\
& e_{\beta} \otimes e_{\gamma}+l x_{\beta} x_{j} e_{\beta} \otimes e_{\gamma} \otimes e_{\gamma}+W^{\prime} .
\end{aligned}
$$

Here, $W^{\prime}$ does contain any of the previous tensors and $M_{i}, N_{i}, P_{i}, M_{\gamma}, N_{\gamma}, P_{\gamma}$ are all nonzeros.

If the coefficient of $e_{\gamma} \otimes e_{\gamma} \otimes e_{\gamma}$ or at least one of the coefficients of $e_{i} \otimes e_{i} \otimes e_{\gamma}, e_{i} \otimes e_{\gamma} \otimes e_{\gamma}, e_{\gamma} \otimes e_{i} \otimes e_{\gamma}, e_{i} \otimes e_{\gamma} \otimes e_{i}, e_{\gamma} \otimes e_{\gamma} \otimes$ $e_{i}, e_{\gamma} \otimes e_{i} \otimes e_{i}$ in $\tau_{\beta}(m)$ is not zero then we refer to cases 1 or 2 and so we are done; otherwise, we consider the following system:

$$
\left\{\begin{array}{c}
a N_{i}+b M_{i}+g M_{i} N_{i}=0 \\
a N_{\gamma}+d P_{\gamma}+g M_{i} N_{\gamma}+h M_{i} P_{\gamma}+l M_{i}=0 \\
b M_{\gamma}+e P_{\gamma}+g M_{\gamma} N_{i}+i N_{i} P_{\gamma}+k N_{i}=0 \\
c M_{i}+d p_{i}+h M_{i} P_{i}=0 \\
c M_{\gamma}+f N_{\gamma}+h M_{\gamma} P_{i}+i N_{\gamma} P_{i}+j P_{i}=0 \\
e P_{i}+f N_{i}+i N_{i} P_{i}=0
\end{array}\right.
$$

Computing $\tau_{\beta}^{2}(m)$, we find that the coefficient of $e_{i} \otimes e_{i} \otimes e_{\gamma}$ is

$$
a y_{\beta} y_{j} N_{i}+b x_{\beta} x_{j} M_{i}+g M_{i} N_{i}\left(y_{\beta} y_{j}+x_{\beta} x_{j}+y_{\beta} y_{j} x_{\beta} x_{j}\right) \text {. }
$$

If this coefficient is nonzero then we refer to Case 2 and so we are done; otherwise, we work with $\tau_{\gamma}(m)$ to get that $a, b, c, d, e, f, g, h, i, j, k \& l$ are all zeros using the system above, which is a contradiction.

Case 4. Suppose that there exist $\alpha, \beta \& \gamma$ different from $i$ such that $e_{\alpha} \otimes e_{\beta} \otimes e_{\gamma} \in \operatorname{supp}(m)$.

We write $m$ as follows:

$$
\begin{aligned}
m= & a e_{\alpha} \otimes e_{\beta} \otimes e_{\gamma}+b e_{\alpha} \otimes e_{\gamma} \otimes e_{\beta}+c e_{\beta} \otimes e_{\alpha} \otimes e_{\gamma}+d e_{\beta} \otimes e_{\gamma} \otimes e_{\alpha}+e e_{\gamma} \otimes e_{\alpha} \otimes \\
& e_{\beta}+f e_{\gamma} \otimes e_{\beta} \otimes e_{\alpha}+W .
\end{aligned}
$$

Here, at least one of $a, b, c, d, e, f \in \mathbb{C}^{*}$ and $\sup p(W)$ does not contain any of the previous tensors. We also assume that $\operatorname{supp}(W)$ does not contain any of $e_{\alpha} \otimes e_{\alpha} \otimes e_{\alpha}$ for any $\alpha$. 
Applying $\tau_{\alpha}$ on $m$, we obtain

$$
\begin{aligned}
\tau_{\alpha}(m)= & a\left(M_{i} e_{i}+x_{\alpha} x_{j} e_{\alpha}+M_{\beta} e_{\beta}+M_{\gamma} e_{\gamma}+\ldots\right) \otimes e_{\beta} \otimes e_{\gamma}+b\left(M_{i} e_{i}+x_{\alpha} x_{j} e_{\alpha}+\right. \\
& \left.M_{\beta} e_{\beta}+M_{\gamma} e_{\gamma}+\ldots\right) \otimes e_{\gamma} \otimes e_{\beta}+c e_{\beta} \otimes\left(N_{i} e_{i}+y_{\alpha} y_{j} e_{\alpha}+N_{\beta} e_{\beta}+N_{\gamma} e_{\gamma}\right. \\
& +\ldots) \otimes e_{\gamma}+d e_{\beta} \otimes e_{\gamma} \otimes\left(P_{i} e_{i}+z_{\alpha} z_{j} e_{\alpha}+P_{\beta} e_{\beta}+P_{\gamma} e_{\gamma}+\ldots\right)+e e_{\gamma} \otimes\left(N_{i}\right. \\
& \left.e_{i}+y_{\alpha} y_{j} e_{\alpha}+N_{\beta} e_{\beta}+N_{\gamma} e_{\gamma}+\ldots\right) \otimes e_{\beta}+f e_{\gamma} \otimes e_{\beta} \otimes\left(P_{i} e_{i}+z_{\alpha} z_{j} e_{\alpha}+P_{\beta}\right. \\
& \left.e_{\beta}+P_{\gamma} e_{\gamma}+\ldots\right)+\tau_{\alpha}(W) \\
= & a M_{i} e_{i} \otimes e_{\beta} \otimes e_{\gamma}+b M_{i} e_{i} \otimes e_{\gamma} \otimes e_{\beta}+c N_{i} e_{\beta} \otimes e_{i} \otimes e_{\gamma}+d P_{i} e_{\beta} \otimes e_{\gamma} \otimes \\
& e_{i}+e N_{i} e_{\gamma} \otimes e_{i} \otimes e_{\beta}+f P_{i} e_{\gamma} \otimes e_{\beta} \otimes e_{i}+\ldots+\tau_{\alpha}(W) .
\end{aligned}
$$

At least one of $a M_{i}, b M_{i}, c N_{i}, d P_{i}, e N_{i}, f P_{i}$ is nonzero. So, by Case 3 , we are done.

Claim 2. $e_{i} \otimes e_{i} \otimes e_{i} \in \operatorname{supp}(m)$ for some $m \in M$.

Proof of Claim 2. We have, by Claim 1, that $e_{s} \otimes e_{s} \otimes e_{s} \in \operatorname{supp}(m)$ for some $s \in\{1, \ldots, n-1\}$ and $m \in M$. We write $m=\alpha_{s} e_{s} \otimes e_{s} \otimes e_{s}+W$ and $\operatorname{supp}(W)$ does not contain $e_{s} \otimes e_{s} \otimes e_{s}$. Here, $\alpha_{s} \in \mathbb{C}^{*}$. It follows that

$$
\begin{aligned}
\tau_{s}(m) & =\alpha_{s} \tau_{s}\left(e_{s} \otimes e_{s} \otimes e_{s}\right)+\tau_{s}(W) \\
& =\alpha_{s}\left(\left(e_{s}-v_{s}\right) \otimes\left(e_{s}-v_{s}\right) \otimes\left(e_{s}-v_{s}\right)\right)+\tau_{s}(W) \\
& =\alpha_{s}\left(\sum_{l=1}^{n-1} A_{l} e_{l} \otimes \sum_{l=1}^{n-1} B_{l} e_{l} \otimes \sum_{l=1}^{n-1} C_{l} e_{l}\right)+\tau_{s}(W) .
\end{aligned}
$$

This implies that $e_{l} \otimes e_{l} \otimes e_{l} \in \operatorname{supp}\left(\tau_{s}(m)\right)$ for every $l \in\{1, \ldots, n-1\}$. In particular, we let $l=i$. Then we get that $e_{i} \otimes e_{i} \otimes e_{i} \in \operatorname{supp}(m), m \in M$.

Claim 3. $v_{i}(x) \otimes v_{i}(y) \otimes v_{i}(z) \in M$.

Proof of Claim 3. A calculation shows that:

$\left(\tau_{i}-x_{i} x_{j} z_{i} z_{j}\right)\left(\tau_{i}-x_{i} x_{j} y_{i} y_{j}\right)\left(\tau_{i}-y_{i} y_{j} z_{i} z_{j}\right)\left(\tau_{i}-z_{i} z_{j}\right)\left(\tau_{i}-y_{i} y_{j}\right)\left(\tau_{i}-x_{i} x_{j}\right)\left(\tau_{i}-1\right)\left(e_{i} \otimes e_{i} \otimes e_{i}\right)$

$=-x_{i}^{3} y_{i}^{3} z_{i}^{3} x_{j}^{3} y_{j}^{3} z_{j}^{3}\left(x_{i} x_{j} y_{i} y_{j}-1\right)\left(x_{i} x_{j} z_{i} z_{j}-1\right)\left(y_{i} y_{j} z_{i} z_{j}-1\right)\left(x_{i} x_{j} y_{i} y_{j} z_{i} z_{j}-1\right)\left(v_{i}(x) \otimes v_{i}(y) \otimes v_{i}(z)\right)$

and

$\left(\tau_{i}-x_{i} x_{j} z_{i} z_{j}\right)\left(\tau_{i}-x_{i} x_{j} y_{i} y_{j}\right)\left(\tau_{i}-y_{i} y_{j} z_{i} z_{j}\right)\left(\tau_{i}-z_{i} z_{j}\right)\left(\tau_{i}-y_{i} y_{j}\right)\left(\tau_{i}-x_{i} x_{j}\right)\left(\tau_{i}-1\right)\left(e_{u} \otimes e_{v} \otimes e_{w}\right)=0$ if $(u, v, w) \neq(i, i, i)$.

Claim 4. For $l \in\{1, \ldots, j-1, j+1, \ldots, n\}$, we have that $v_{l} \otimes v_{l} \otimes v_{l} \in M$.

Proof of Claim 4. We have, by claim 3, that $v_{i}(x) \otimes v_{i}(y) \otimes v_{i}(z) \in M$. Here, $v_{i}=v_{i, j}$. Applying Lemma 2, we have that $f_{i, j}\left(v_{i} \otimes v_{i} \otimes v_{i}\right) \in M$, which implies that $v_{i+1} \otimes v_{i+1} \otimes v_{i+1} \in M$. Similarly, we also have that $g_{i, j}\left(v_{i} \otimes v_{i} \otimes v_{i}\right) \in M$, which implies that $v_{i-1} \otimes v_{i-1} \otimes v_{i-1} \in M$. After a consecuitive use of $f_{i, j}, f_{i+1, j}, \ldots$ and $g_{i, j}, g_{i-1, j}, \ldots$, we obtain that

$$
v_{l} \otimes v_{l} \otimes v_{l} \in M \text { for every } l \in\{1, \ldots, j-1, j+1, \ldots, n\} .
$$

Claim 5. For $p, q, r \in\{1, \ldots, j-1, j+1, \ldots, n\}, v_{p} \otimes v_{q} \otimes v_{r} \in M$.

Proof of Claim 5. We consider the following cases:

Case 5. $p=q=r$, we are done.

Case 6. $p=q=i, r \neq i$.

Applying $\tau_{i}$ on $v_{r} \otimes v_{r} \otimes v_{r} \in M$, we obtain $\left(v_{r}+a v_{i}\right) \otimes\left(v_{r}+b v_{i}\right) \otimes\left(v_{r}+c v_{i}\right) \in M$, and so

$$
\begin{gathered}
c v_{r} \otimes v_{r} \otimes v_{i}+b v_{r} \otimes v_{i} \otimes v_{r}+b c v_{r} \otimes v_{i} \otimes v_{i}+a v_{i} \otimes v_{r} \otimes v_{r}+a c v_{i} \otimes v_{r} \otimes v_{i}+ \\
a b v_{i} \otimes v_{i} \otimes v_{r} \in M .
\end{gathered}
$$

Applying $\tau_{i}$ again, we obtain

$$
\begin{gathered}
c z_{i} z_{j} v_{r} \otimes v_{r} \otimes v_{i}+b y_{i} y_{j} v_{r} \otimes v_{i} \otimes v_{r}+a x_{i} x_{j} v_{i} \otimes v_{r} \otimes v_{r}+a b\left(x_{i} x_{j}+y_{i} y_{j}+x_{i} x_{j} y_{i} y_{j}\right) v_{i} \otimes v_{i} \otimes v_{r} \quad+ \\
a c\left(x_{i} x_{j}+z_{i} z_{j}+x_{i} x_{j} z_{i} z_{j}\right) v_{i} \otimes v_{r} \otimes v_{i}+b c\left(y_{i} y_{j}+z_{i} z_{j}+y_{i} y_{j} z_{i} z_{j}\right) v_{r} \otimes v_{i} \otimes v_{i} \in M .
\end{gathered}
$$

Combining (1) and (2), we get 
$\left(y_{i} y_{j}-z_{i} z_{j}\right) b v_{r} \otimes v_{i} \otimes v_{r}+\left(x_{i} x_{j}-z_{i} z_{j}\right) a v_{i} \otimes v_{r} \otimes v_{r}+\left(x_{i} x_{j}+y_{i} y_{j}-z_{i} z_{j}+x_{i} x_{j} y_{i} y_{j}\right) a b v_{i} \otimes v_{i} \otimes v_{r}+$ $\left(x_{i} x_{j}+x_{i} x_{j} z_{i} z_{j}\right) a c v_{i} \otimes v_{r} \otimes v_{i}+\left(y_{i} y_{j}+y_{i} y_{j} z_{i} z_{j}\right) b c v_{r} \otimes v_{i} \otimes v_{i} \in M$.

Applying $\tau_{i}$ again, we obtain

$$
\begin{gathered}
y_{i} y_{j}\left(y_{i} y_{j}-z_{i} z_{j}\right) b v_{r} \otimes v_{i} \otimes v_{r}+x_{i} x_{j}\left(x_{i} x_{j}-z_{i} z_{j}\right) a v_{i} \otimes v_{r} \otimes v_{r}+ \\
a b\left[\left(x_{i} x_{j}+y_{i} y_{j}-z_{i} z_{j}+x_{i} x_{j} y_{i} y_{j}\right) x_{i} x_{j} y_{i} y_{j}+y_{i} y_{j}\left(y_{i} y_{j}-z_{i} z_{j}\right)+x_{i} x_{j}\left(x_{i} x_{j}-z_{i} z_{j}\right)\right] v_{i} \otimes v_{i} \otimes v_{r}+ \\
a c\left[x_{i} x_{j}\left(x_{i} x_{j}-z_{i} z_{j}\right)+\left(x_{i} x_{j}+x_{i} x_{j} z_{i} z_{j}\right) x_{i} x_{j} z_{i} z_{j}\right] v_{i} \otimes v_{r} \otimes v_{i}+ \\
b c\left[y_{i} y_{j}\left(y_{i} y_{j}-z_{i} z_{j}\right)+\left(y_{i} y_{j}+y_{i} y_{j} z_{i} z_{j}\right) y_{i} y_{j} z_{i} z_{j}\right] v_{r} \otimes v_{i} \otimes v_{i} \in M .
\end{gathered}
$$

Combining (3) and (4), we let

$\alpha=\left[a\left(x_{i} x_{j}-z_{i} z_{j}\right)\left(x_{i} x_{j}-y_{i} y_{j}\right)\right], \beta=\left[a b\left(x_{i}^{2} x_{j}^{2} y_{i} y_{j}-x_{i} x_{j} y_{i} y_{j} z_{i} z_{j}+x_{i}^{2} x_{j}^{2} y_{i}^{2} y_{j}^{2}+x_{i}^{2} x_{j}^{2}-x_{i} x_{j} z_{i} z_{j}-x_{i} x_{j} y_{i} y_{j}\right)\right], \gamma=\left[a c\left(x_{i}^{2} x_{j}^{2}-\right.\right.$ $\left.\left.x_{i} x_{j} z_{i} z_{j}+x_{i}^{2} x_{j}^{2} z_{i} z_{j}+x_{i}^{2} x_{j}^{2} z_{i}^{2} z_{j}^{2}-x_{i} x_{j} y_{i} y_{j}-x_{i} x_{j} y_{i} y_{j} z_{i} z_{j}\right)\right], \delta=\left[b c\left(-y_{i} y_{j} z_{i} z_{j}+y_{i}^{2} y_{j}^{2} z_{i}^{2} z_{j}^{2}\right)\right]$

then

$$
\alpha v_{i} \otimes v_{r} \otimes v_{r}+\beta v_{i} \otimes v_{i} \otimes v_{r}+\gamma v_{i} \otimes v_{r} \otimes v_{i}+\delta v_{r} \otimes v_{i} \otimes v_{i} \in M
$$

Applying $\tau_{i}$ again and simplifying, we obtain

$$
\begin{gathered}
\alpha x_{i} x_{j} v_{i} \otimes v_{r} \otimes v_{r}+\left(\alpha x_{i} x_{j} c+\gamma x_{i} x_{j} z_{i} z_{j}\right) v_{i} \otimes v_{r} \otimes v_{i}+ \\
\left(\alpha x_{i} x_{j} b+\beta x_{i} x_{j} y_{i} y_{j}\right) v_{i} \otimes v_{i} \otimes v_{r}+\delta y_{i} y_{j} z_{i} z_{j} v_{r} \otimes v_{i} \otimes v_{i} \in M .
\end{gathered}
$$

Combining (5) and (6), we get

$$
\begin{gathered}
\left(\alpha x_{i} x_{j} b+\beta x_{i} x_{j} y_{i} y_{j}-\beta x_{i} x_{j}\right) v_{i} \otimes v_{i} \otimes v_{r}+\left(\alpha x_{i} x_{j} c+\gamma x_{i} x_{j} z_{i} z_{j}-\gamma x_{i} x_{j}\right) v_{i} \otimes v_{r} \otimes v_{i}+ \\
\left(\delta y_{i} y_{j} z_{i} z_{j}-\delta x_{i} x_{j}\right) v_{r} \otimes v_{i} \otimes v_{i} \in M
\end{gathered}
$$

Applying $\tau_{i}$ again and simplifying, we obtain

$$
\begin{gathered}
\left(\alpha x_{i} x_{j} b+\beta x_{i} x_{j} y_{i} y_{j}-\beta x_{i} x_{j}\right) x_{i} x_{j} y_{i} y_{j} v_{i} \otimes v_{i} \otimes v_{r}+\left(\alpha x_{i} x_{j} c+\gamma x_{i} x_{j} z_{i} z_{j}-\gamma x_{i} x_{j}\right) \\
x_{i} x_{j} z_{i} z_{j} v_{i} \otimes v_{r} \otimes v_{i}+\left(\delta y_{i} y_{j} z_{i} z_{j}-\delta x_{i} x_{j}\right) y_{i} y_{j} z_{i} z_{j} v_{r} \otimes v_{i} \otimes v_{i} \in M .
\end{gathered}
$$

Combining (7) and (8), we get

$$
\begin{gathered}
\left(\alpha x_{i} x_{j} b+\beta x_{i} x_{j} y_{i} y_{j}-\beta x_{i} x_{j}\right)\left(x_{i} x_{j} y_{i} y_{j}-y_{i} y_{j} z_{i} z_{j}\right) v_{i} \otimes v_{i} \otimes v_{r}+ \\
\left(\alpha x_{i} x_{j} c+\gamma x_{i} x_{j} z_{i} z_{j}-\gamma x_{i} x_{j}\right)\left(x_{i} x_{j} z_{i} z_{j}-y_{i} y_{j} z_{i} z_{j}\right) v_{i} \otimes v_{r} \otimes v_{i} \in M
\end{gathered}
$$

Applying $\tau_{i}$ again and simplifying, we obtain

$$
\begin{gathered}
\left(\alpha x_{i} x_{j} b+\beta x_{i} x_{j} y_{i} y_{j}-\beta x_{i} x_{j}\right)\left(x_{i} x_{j} y_{i} y_{j}-y_{i} y_{j} z_{i} z_{j}\right) x_{i} x_{j} y_{i} y_{j} v_{i} \otimes v_{i} \otimes v_{r}+ \\
\left(\alpha x_{i} x_{j} c+\gamma x_{i} x_{j} z_{i} z_{j}-\gamma x_{i} x_{j}\right)\left(x_{i} x_{j} z_{i} z_{j}-y_{i} y_{j} z_{i} z_{j}\right) x_{i} x_{j} z_{i} z_{j} v_{i} \otimes v_{r} \otimes v_{i} \in M .
\end{gathered}
$$

Combining (9) and (10), we get

$\left(\alpha x_{i} x_{j} b+\beta x_{i} x_{j} y_{i} y_{j}-\beta x_{i} x_{j}\right)\left(x_{i} x_{j} y_{i} y_{j}-y_{i} y_{j} z_{i} z_{j}\right)\left(x_{i} x_{j} y_{i} y_{j}-x_{i} x_{j} z_{i} z_{j}\right) v_{i} \otimes v_{i} \otimes v_{r} \in M$.

Since $\left(\alpha x_{i} x_{j} b+\beta x_{i} x_{j} y_{i} y_{j}-\beta x_{i} x_{j}\right)\left(x_{i} x_{j} y_{i} y_{j}-y_{i} y_{j} z_{i} z_{j}\right)\left(x_{i} x_{j} y_{i} y_{j}-x_{i} x_{j} z_{i} z_{j}\right)=a b x_{i}^{2} x_{j}^{2} y_{i}^{2} y_{j}^{2}\left(x_{i} x_{j}-z_{i} z_{j}\right)\left(y_{i} y_{j}-z_{i} z_{j}\right)\left(x_{i} x_{j} y_{i} y_{j}-\right.$ 1) $\left(x_{i} x_{j} y_{i} y_{j}-z_{i} z_{j}\right) \neq 0$, it follows that $v_{i} \otimes v_{i} \otimes v_{r} \in M$.

Similarly, we can prove that $v_{i} \otimes v_{q} \otimes v_{i}$ and $v_{p} \otimes v_{i} \otimes v_{i} \in M$ since $a c x_{i}^{2} x_{j}^{2} z_{i}^{2} z_{j}^{2}\left(y_{i} y_{j}-z_{i} z_{j}\right)\left(x_{i} x_{j}-y_{i} y_{j}\right)\left(x_{i} x_{j} z_{i} z_{j}-1\right)\left(x_{i} x_{j} z_{i} z_{j}-\right.$ $\left.y_{i} y_{j}\right) \neq 0$ and $b c y_{i}^{2} y_{j}^{2} z_{i}^{2} z_{j}^{2}\left(x_{i} x_{j}-z_{i} z_{j}\right)\left(x_{i} x_{j}-y_{i} y_{j}\right)\left(y_{i} y_{j} z_{i} z_{j}-1\right)\left(y_{i} y_{j} z_{i} z_{j}-x_{i} x_{j}\right) \neq 0$.

Case 7. $p=q \neq i, r=i$.

Applying $\tau_{p}$ on $v_{i} \otimes v_{i} \otimes v_{i} \in M$, we obtain

$$
b c v_{i} \otimes v_{p} \otimes v_{p}+a c v_{p} \otimes v_{i} \otimes v_{p}+a b v_{p} \otimes v_{p} \otimes v_{i} \in M
$$


Applying $\tau_{i}$ and simplifying, we obtain

$$
b c x_{i} x_{j} v_{i} \otimes v_{p} \otimes v_{p}+a c y_{i} y_{j} v_{p} \otimes v_{i} \otimes v_{p}+a b z_{i} z_{j} v_{p} \otimes v_{p} \otimes v_{i} \in M .
$$

Combining (11) and (12), we get

$$
a c\left(y_{i} y_{j}-x_{i} x_{j}\right) v_{p} \otimes v_{i} \otimes v_{p}+a b\left(z_{i} z_{j}-x_{i} x_{j}\right) v_{p} \otimes v_{p} \otimes v_{i} \in M .
$$

Applying $\tau_{i}$ again and simplifying, we obtain

$$
a c y_{i} y_{j}\left(y_{i} y_{j}-x_{i} x_{j}\right) v_{p} \otimes v_{i} \otimes v_{p}+a b z_{i} z_{j}\left(z_{i} z_{j}-x_{i} x_{j}\right) v_{p} \otimes v_{p} \otimes v_{i} \in M .
$$

Combining (13) and (14), we get

$$
a b\left(z_{i} z_{j}-x_{i} x_{j}\right)\left(z_{i} z_{j}-y_{i} y_{j}\right) v_{p} \otimes v_{p} \otimes v_{i} \in M .
$$

Since $a b\left(z_{i} z_{j}-x_{i} x_{j}\right)\left(z_{i} z_{j}-y_{i} y_{j}\right) \neq 0$, it follows that $v_{p} \otimes v_{p} \otimes v_{i} \in M$. Similarly, we prove that $v_{p} \otimes v_{i} \otimes v_{p}$ and $v_{i} \otimes v_{q} \otimes v_{q} \in M$ since $a c\left(y_{i} y_{j}-x_{i} x_{j}\right)\left(y_{i} y_{j}-z_{i} z_{j}\right) \neq 0$ and $b c\left(x_{i} x_{j}-y_{i} y_{j}\right)\left(x_{i} x_{j}-z_{i} z_{j}\right) \neq 0$.

Case 8. $p \neq q \neq i, r=i$.

Applying $\tau_{p}$ on $v_{i} \otimes v_{q} \otimes v_{i} \in M$, we obtain

$$
\left(v_{i}+a v_{p}\right) \otimes\left(v_{q}+b^{\prime} v_{p}\right) \otimes\left(v_{i}+c v_{p}\right) \in M .
$$

Simplifying, we obtain

$$
c v_{i} \otimes v_{q} \otimes v_{p}+a v_{p} \otimes v_{q} \otimes v_{i}+a c v_{p} \otimes v_{q} \otimes v_{p} \in M .
$$

Applying $\tau_{i}$ again and simplifying, we obtain

$$
c\left(x_{i} x_{j}+a a^{\prime \prime \prime}\right) v_{i} \otimes v_{q} \otimes v_{p}+a\left(z_{i} z_{j}+c c^{\prime \prime \prime}\right) v_{p} \otimes v_{q} \otimes v_{i}+a c v_{p} \otimes v_{q} \otimes v_{p} \in M .
$$

Combining (15) and (16), we get

$$
c\left(x_{i} x_{j}+a a^{\prime \prime \prime}-1\right) v_{i} \otimes v_{q} \otimes v_{p}+a\left(z_{i} z_{j}+c c^{\prime \prime \prime}-1\right) v_{p} \otimes v_{q} \otimes v_{i} \in M .
$$

Applying $\tau_{i}$ and simplifying, we obtain

$$
c x_{i} x_{j}\left(x_{i} x_{j}+a a^{\prime \prime \prime}-1\right) v_{i} \otimes v_{q} \otimes v_{p}+a z_{i} z_{j}\left(z_{i} z_{j}+c c^{\prime \prime \prime}-1\right) v_{p} \otimes v_{q} \otimes v_{i} \in M .
$$

Combining (17) and (18), we get

$$
a\left(z_{i} z_{j}-x_{i} x_{j}\right)\left(z_{i} z_{j}+c c^{\prime \prime \prime}-1\right) v_{p} \otimes v_{q} \otimes v_{i} \in M .
$$

So, $v_{p} \otimes v_{q} \otimes v_{i} \in M$ since $a\left(z_{i} z_{j}-x_{i} x_{j}\right)\left(z_{i} z_{j}+c c^{\prime \prime \prime}-1\right) \neq 0$.

Note that if $z_{i} z_{j}+c c^{\prime \prime \prime}-1=0$, then this contradicts $x_{i} \neq x_{j}, y_{i} \neq y_{j}, z_{i} \neq z_{j}$. Similarly, we can prove that $v_{p} \otimes v_{i} \otimes v_{r}$ and $v_{i} \otimes v_{q} \otimes v_{r} \in M$.

Case 9. $p=q, \quad p \neq i, q \neq i$ and $r \neq i$

Applying $\tau_{p}$ on $v_{i} \otimes v_{i} \otimes v_{r} \in M$, we obtain

$$
\left(v_{i}+a v_{p}\right) \otimes\left(v_{i}+b v_{p}\right) \otimes\left(v_{r}+c^{\prime} v_{p}\right) \in M .
$$

Simplifying, we get that $a b v_{p} \otimes v_{p} \otimes v_{r} \in M$ and so $v_{p} \otimes v_{p} \otimes v_{r} \in M$. Also, we prove that $v_{p} \otimes v_{q} \otimes v_{p}$ and $v_{p} \otimes v_{q} \otimes v_{q} \in M$.

Case 10. $p, q$ and $r$ are different from $i, p \neq q, p \neq r, q \neq r$.

Applying $\tau_{r}$ on $v_{p} \otimes v_{q} \otimes v_{i} \in M$, we obtain

$$
\left(v_{p}+a v_{r}\right) \otimes\left(v_{q}+b^{\prime} v_{r}\right) \otimes\left(v_{i}+c^{\prime \prime} v_{r}\right) \in M .
$$

Simplifying, we get that $c^{\prime \prime} v_{p} \otimes v_{q} \otimes v_{r} \in M$ and so $v_{p} \otimes v_{q} \otimes v_{r} \in M$. 
Consider the representation $G_{n}\left(t_{1}, \ldots, t_{n}\right): P_{n} \rightarrow G L_{n-1}\left(\mathbb{C}\left[t_{1}{ }^{ \pm 1}, \ldots, t_{n}{ }^{ \pm 1}\right]\right)$,

where $t_{1}, \ldots, t_{n}$ are indeterminates. Specializing $t_{1}, \ldots, t_{n}$ to nonzero complex numbers $x_{1}, \ldots, x_{n}$ defines a representation $G_{n}\left(x_{1}, \ldots, x_{n}\right): U_{j} \rightarrow G L_{n-1}(\mathbb{C})=G L\left(\mathbb{C}^{n-1}\right)$ which is irreducible if and only if $q\left(x_{1}, \ldots, x_{n}\right)=x_{1} \ldots x_{n}-1 \neq 0$. Next, we get our main theorem.

Theorem 1. For $n \geq 3$, consider the tensor product of irreducible representations $G_{n}^{(1)}\left(x_{1}, \ldots, x_{n}\right) \otimes G_{n}^{(2)}\left(y_{1}, \ldots, y_{n}\right) \otimes$ $G_{n}^{(3)}\left(z_{1}, \ldots, z_{n}\right): U_{j} \rightarrow G L\left(\mathbb{C}^{n-1} \otimes \mathbb{C}^{n-1} \otimes \mathbb{C}^{n-1}\right)$, where $q\left(x_{1}, \ldots, x_{n}\right) \neq 0, q\left(y_{1}, \ldots, y_{n}\right) \neq 0$ and $q\left(z_{1}, \ldots, z_{n}\right) \neq 0$. If for some $i \neq j, x_{i} x_{j} \neq y_{i} y_{j}, x_{i} x_{j} \neq z_{i} z_{j}, y_{i} y_{j} \neq z_{i} z_{j}, x_{i} x_{j} y_{i} y_{j} \neq 1, x_{i} x_{j} z_{i} z_{j} \neq 1, y_{i} y_{j} z_{i} z_{j} \neq 1, x_{i} x_{j} \neq y_{i} y_{j} z_{i} z_{j}, y_{i} y_{j} \neq x_{i} x_{j} z_{i} z_{j}, z_{i} z_{j} \neq$ $x_{i} x_{j} y_{i} y_{j}, x_{i} x_{j} y_{i} y_{j} z_{i} z_{j} \neq 1, x_{i} \neq x_{j}, y_{i} \neq y_{j}, z_{i} \neq z_{j}$, then the above representation is irreducible.

Proof. By Proposition 1, we have that $\mathcal{A} \mathbb{C}^{n-1} \otimes \mathcal{A} \mathbb{C}^{n-1} \otimes \mathcal{A} \mathbb{C}^{n-1}$ is the unique minimal non zero $U_{j}$-submodule of $\mathbb{C}^{n-1} \otimes \mathbb{C}^{n-1} \otimes \mathbb{C}^{n-1}$. In particular, it is an irreducible $U_{j}$-module. The fact that $q\left(x_{1}, \ldots, x_{n}\right) \neq 0, q\left(y_{1}, \ldots, y_{n}\right) \neq 0$ and $q\left(z_{1}, \ldots, z_{n}\right) \neq 0$ implies that the first factor $\mathcal{A} \mathbb{C}^{n-1}$ corresponds to the representation $G_{n}^{(1)}\left(x_{1}, \ldots, x_{n}\right)$, the second factor $\mathcal{A} \mathbb{C}^{n-1}$ corresponds to the representation $G_{n}^{(2)}\left(y_{1}, \ldots, y_{n}\right)$ and the third factor $\mathcal{A} \mathbb{C}^{n-1}$ corresponds to the representation $G_{n}^{(3)}\left(z_{1}, \ldots, z_{n}\right) \quad \square$.

Since irreducibility on a subgroup implies irreducibility on the group itself, it follows that Theorem 1 is also true for the tensor product of specializations of the Gassner representation of the pure braid group.

\section{The Tensor Product oF $k$ Irreducible Gassner Representations ( $k \geq 3)$}

We now introduce Proposition 2 that provides us with a sufficient condition for the irreducibility of the tensor product of $k$ irreducible Gassner representations $G_{n}^{(1)}\left(x_{11}, \ldots, x_{n 1}\right) \otimes \ldots \otimes G_{n}^{(k)}\left(x_{1 k}, \ldots, x_{n k}\right): U_{j} \rightarrow G L\left(\mathbb{C}^{n-1} \otimes \ldots \otimes \mathbb{C}^{n-1}\right)$, where $n \geq 3$ and $k \geq 3$.

Proposition 2. Given $k \geq 3$ and $x_{1}=\left(x_{11}, \ldots, x_{n 1}\right), \ldots, x_{k}=\left(x_{1 k}, \ldots, x_{n k}\right) \in \mathbb{C}^{n}$, where $x_{r s} \in \mathbb{C}-\{0,1\}$. Here, $1 \leq r \leq$ $n$ and $1 \leq s \leq k$. Suppose that for some $i<j$ and every integer $s$ with $1 \leq s \leq k$, we have that

$$
x_{i s} x_{j s} \neq\left(\prod_{\substack{\alpha=1 \\ l_{\alpha} \neq s}}^{\substack{m \leq k-1 \\ m}} x_{i l_{\alpha}} x_{j l_{\alpha}}\right)^{ \pm 1} \text { and } x_{i s} \neq x_{j s}
$$

(We might have the same condition repeated more than once. Here, $1 \leq \alpha \leq k-1,1 \leq l_{\alpha} \leq k$ and $l_{\alpha}$ 's are taken to be distinct for different values of $\alpha$.)

Let $M$ be a nonzero $U_{j}$-submodule of $\mathbb{C}^{n-1} \otimes \ldots \otimes \mathbb{C}^{n-1}$ under the action of $G_{n}^{(1)}\left(x_{11}, \ldots, x_{n 1}\right) \otimes \ldots \otimes G_{n}^{(k)}\left(x_{1 k}, \ldots, x_{n k}\right): U_{j} \rightarrow$ $G L\left(\mathbb{C}^{n-1} \otimes \ldots \otimes \mathbb{C}^{n-1}\right)$, where $n \geq 3$. For simplicity, we write $v_{p, j}=v_{p}$ for $p \in\{1, \ldots, j-1, j+1, \ldots, n\}$. Then $M$ contains all $v_{p_{1}}\left(x_{1}\right) \otimes \ldots \otimes v_{p_{k}}\left(x_{k}\right)$, where $p_{1}, \ldots, p_{k} \in\{1, \ldots, k\}$. Thus $M$ contains $\mathcal{A} \mathbb{C}^{n-1} \otimes \ldots \otimes \mathcal{A} \mathbb{C}^{n-1}$. Here, the action of $U_{j}$ on the first factor is induced by $G_{n}^{(1)}\left(x_{11}, \ldots, x_{n 1}\right), \ldots$, the action of $U_{j}$ on the last factor is induced by $G_{n}^{(k)}\left(x_{1 k}, \ldots, x_{n k}\right)$.

Proof. For $1 \leq j \leq n$, we consider the normal free subgroup of rank $n-1$, namely, $U_{j}$. Almost the same proof, as in the case $k=3$, is applied here. However, in the general case, we have a lot of tidious computations with large matrices and a lot of cases to handle. We will not repeat the argument, but we will rather generalize some of the crucial matrix forms and equations used in section three.

To generalize Case 2 of Claim 1 in section 3, we write $m$ along the same lines as before and we apply $\tau_{i j}, \tau_{i j}^{2}, \ldots, \tau_{i j}^{2^{k}-2}$ on $m$. If one of the coefficients of $e_{s} \otimes \ldots \otimes e_{s}$ is nonzero, then we are done; otherwise, we consider the $\left(2^{k}-2\right) \times\left(2^{k}-2\right)$ matrix $A=\left[a_{\alpha \beta}\right]$ defined as follows:

$$
\left(\begin{array}{cccccccc}
a_{1,1} & \cdots & a_{1, k} & a_{1,1} \cdot a_{1,2} & \cdots & a_{1, k-1} \cdot a_{1, k} & \cdots & a_{1,2} \ldots a_{1, k} \\
a_{2,1} & \cdots & a_{2, k} & a_{2,1} \cdot a_{2,2} & \cdots & a_{2, k-1} \cdot a_{2, k} & \cdots & a_{2,2} \ldots a_{2, k} \\
\vdots & \ldots & \vdots & \vdots & \ldots & \vdots & \ldots & \vdots \\
a_{2^{k}-2,1} & \cdots & a_{2^{k}-2, k} & a_{2^{k}-2,1} \cdot a_{2^{k}-2,2} & \cdots & a_{2^{k}-2, k-1} \cdot a_{2^{k}-2, k} & \cdots & a_{2^{k}-2,2} \ldots a_{2^{k}-2, k}
\end{array}\right)
$$

The entries in the matrix $A$ are given by

$$
a_{\alpha, \beta}=M_{s \beta}\left(\sum_{k=0}^{\alpha-1} x_{i \beta}^{k} x_{j \beta}^{k}\right), \quad 1 \leq \alpha \leq 2^{k}-2, \quad 1 \leq \beta \leq k .
$$


Also the non zero complex numbers $M_{s \beta}$ 's are given as follows:

If $s<i$ then $M_{s \beta}=x_{s \beta}-1$.

If $i<s<j$ then $M_{s \beta}=x_{j \beta}\left(x_{s \beta}-1\right)$.

If $s \geq j$ then $M_{s \beta}=1-x_{s \beta}$.

Claim 3 of section three is generalized as follows:

$$
A\left(e_{i} \otimes \ldots \otimes e_{i}\right)=\alpha_{i j}\left(v_{i}\left(x_{1}\right) \otimes \ldots \otimes v_{i}\left(x_{k}\right)\right)
$$

and

$$
A\left(e_{l_{1}} \otimes \ldots \otimes e_{l_{k}}\right)=0 \quad \text { if } \quad\left(l_{1}, \ldots, l_{k}\right) \neq(i, \ldots, i)
$$

Here the pure braid element $A$ of $U_{j}$, in the case $k \geq 3$, is given by

$$
A=\left(\tau_{i}-1\right) \prod_{m=1}^{k-1} \prod_{\alpha_{1}, \ldots, \alpha_{m} \in\{1, \ldots, k\}}\left[\tau_{i}-\left(x_{i \alpha_{1}} x_{j \alpha_{1}}\right) \ldots\left(x_{i \alpha_{m}} x_{j \alpha_{m}}\right)\right]
$$

For a fixed integer $m$, we have $C_{m}^{k}$ possible products for $\left[\tau_{i}-\left(x_{i \alpha_{1}} x_{j \alpha_{1}}\right) \ldots\left(x_{i \alpha_{m}} x_{j \alpha_{m}}\right)\right]$ because $\alpha_{1}, \ldots, \alpha_{m}$ are $m$ different integers in $\{1, \ldots, k\}$. Here $C_{m}^{k}$ denotes the number of m-combinations from a set of $k$ objects.

Since irreducibility on $U_{j}$ implies irreducibility on $P_{n}$, we get a similar theorem to that of section 3 .

Theorem 2. For $n \geq 3$ and $k \geq 3$, consider the tensor product of irreducible representations $G_{n}^{(1)}\left(x_{11}, \ldots, x_{n 1}\right) \otimes \ldots \otimes$ $G_{n}^{(k)}\left(x_{1 k}, \ldots, x_{n k}\right): P_{n} \rightarrow G L\left(\mathbb{C}^{n-1} \otimes \ldots \otimes \mathbb{C}^{n-1}\right)$, where $q\left(x_{11}, \ldots, x_{n 1}\right) \neq 0, \ldots, q\left(x_{1 k}, \ldots, x_{n k}\right) \neq 0$. If for some $i \neq j$ and every integer $s$ with $1 \leq s \leq k$, we have that

$$
x_{i s} x_{j s} \neq\left(\prod_{\substack{\alpha=1 \\ l_{\alpha} \neq s}}^{\substack{m \leqslant k-1 \\ m}} x_{i l_{\alpha}} x_{j l_{\alpha}}\right)^{ \pm 1} \text { and } x_{i s} \neq x_{j s}
$$

then the above representation is irreducible. (We might have the same condition repeated more than once)

\section{References}

Abdulrahim, M. (2009).Tensor Products of the Gassner Representation of the Pure Braid Group. Open Math J., 2, 12-15. http://dx.doi.org/10.2174/1874117400902010012

Abdulrahim, M. (2010). On the Irreducibility of Linear Representations of the Pure Braid Group. Tamkang Journal of Mathematics, 41(3), 283-292. http://dx.doi.org/10.5556/j.tkjm.41.2010.283-292

Abdulrahim, M. \& Formanek, E. (2005). Tensor Products of Specializations of the Burau Representation. J.Pure Appl.Algebra, 203(1-3), 104-112. http://dx.doi.org/10.1016/j.jpaa.2005.02.005

Birman, J. S. (1975).Braids, Links and Mapping Class Groups. Annals of Mathematical Studies 82, Princeton University Press, New Jersey, ISBN: 0691081492.

Formanek, E. (1996). Braid group representations of low degree. Proc. London Math. Soc., 73, 279-322. http://dx.doi.org/ 10.1112/plms/s3-73.2.279 